\title{
Erratum to: Neuroprotection by Chlorpromazine and Promethazine in Severe Transient and Permanent Ischemic Stroke
}

\author{
Xiaokun Geng ${ }^{1,2} \cdot$ Fengwu $\mathrm{Li}^{1}$ • James Yip ${ }^{2}$ Changya Peng ${ }^{2}$ • Omar Elmadhoun ${ }^{2}$. \\ Jiamei Shen ${ }^{1} \cdot$ Xunming $\mathrm{Ji}^{1,3} \cdot$ Yuchuan Ding ${ }^{1,2}$
}

Published online: 28 June 2017

(C) Springer Science+Business Media, LLC 2017

Erratum to: Mol Neurobiol

DOI 10.1007/s12035-016-0280-x

Unfortunately, the online published article has mistake in Fig. $7 b$.

The same Western blot images were accidentally placed by authors for both proteins. With this, the authors hereby published the below corrected Fig. 7.

The online version of the original article can be found at http://dx.doi.org/10. 1007/s12035-016-0280-x

Xunming Ji

jixm@ccmu.edu.cn

$\triangle$ Yuchuan Ding

yding@med.wayne.edu

1 China-America Institute of Neuroscience, Beijing Luhe Hospital, Capital Medical University, Beijing 101100, China

2 Department of Neurosurgery, Wayne State University School of Medicine, 550 E Canfield, Detroit, MI 48201, USA

3 Department of Neurosurgery, Xuanwu Hospital, Capital Medical University, Beijing, China
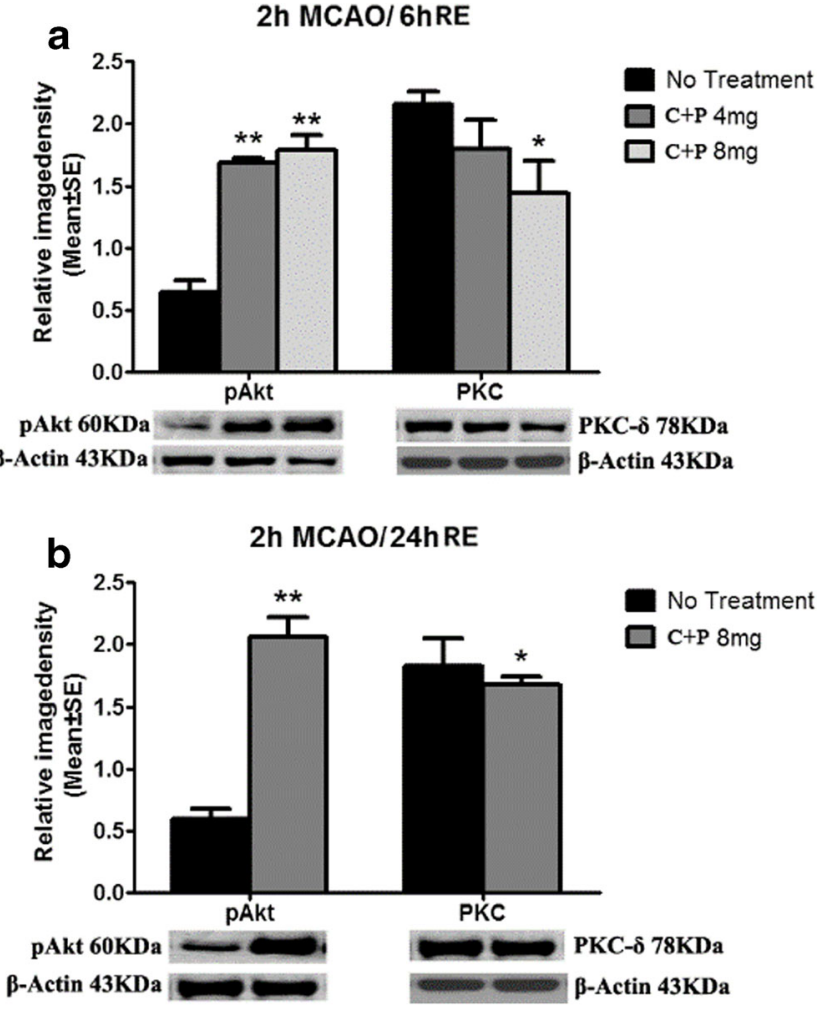